\title{
Relationship of the Duration and Timing of Exercise with Sleep Quality in Community-Dwelling Adults
}

\author{
Sun Hee Lee, MD¹, Seong Jae Kim, MD², Jin Woo Bang, BA ${ }^{1}$, Jung Hie Lee, MD, PhD ${ }^{1,3}$ \\ 'Department of Psychiatry, Kangwon National University Hospital, Chuncheon, Korea \\ 2Department of Psychiatry, Doeun Hospital, Jincheon, Korea \\ ${ }^{3}$ Deparment of Psychiatry, Kangwon National University School of Medicine, Chuncheon, Korea
}

Received: September 7, 2018

Revised: October 9, 2018

Accepted: October 15, 2018

\section{Correspondence}

Jung Hie Lee, MD, PhD

Department of Psychiatry,

Kangwon National University Hospital,

156 Baengnyeong-ro,

Chuncheon 24289, Korea

Tel +82-33-258-2310

Fax +82-33-256-3344

E-mail jhielee@kangwon.ac.kr

ORCID

Sun Hee Lee

https://orcid.org/0000-0003-0393-7360

Seong Jae Kim

https://orcid.org/0000-0003-1699-9560

Jin Woo Bang

https://orcid.org/0000-0003-1851-8695

Jung Hie Lee

https://orcid.org/0000-0002-1471-2602
Background and Objective We aimed to compare the differences in the subjective and objective sleep quality, and quality of life (QOL) according to the duration and timing of exercise in community-dwelling adults, and to compare these between the exercise group (EG) and non-exercise group (non-EG) in insomnia patients.

Methods We recruited 223 volunteers (EG: $\mathrm{n}=119$, age: $60.8 \pm 12.8$ years; non-EG: $\mathrm{n}=104$, age $61.6 \pm 13.3$ years), who visited to 3 Public Health Centers in a rural area of South Korea. The Pittsburgh Sleep Quality Index (PSQI), Korean version of Epworth Sleepiness Scale (KESS), and Short Form-12 Health Survey Questionnaire (SF-12) were administered for each subject. Actigraphy (Actiwatch-2, Philips Respironics Co.) recording was done for 7 days at home, and we included the data of 183 subjects in our analysis. We compared the scores of questionnaires and objective sleep parameters according to the duration and timing of exercise, and compared these between the EG and non-EG in insomnia patients.

Results Physical component summary (PCS) scores in SF-12 were higher in the EG for more than 60 minutes per day. In the subjects with outdoor exercise, the afternoon EG had lower PSQI scores and higher KESS scores. In insomnia patients, PCS scores in the EG was higher than those of the non-EG.

Conclusions Community-dwelling adults who exercised for more than one hour showed higher physical QOL compared to those for less than one hour. Insomnia patients who exercised also showed higher physical QOL. In outdoor exercise, afternoon exercise would be beneficial for subjective sleep quality than morning exercise.

Sleep Med Res 2018;9(2):83-91

Key Words Exercise, Sleep quality, Quality of life, Insomnia.

\section{INTRODUCTION}

Insomnia is the most common sleep disorder, and its prevalence has been reported between $9 \%$ and $15 \%$ of the general population [1]. Insomnia often occurs with various psychiatric and medical disorders including depression, drug abuse and hypertension. It is also known to be a risk factor for those conditions [2]. It has been reported that daytime consequences of insomnia led to a worsening quality of life (QOL), and social and occupational impairments [3]. Given these facts, providing an active treatment for insomnia patients would be clinically crucial.

Both of objective and subjective sleep quality in insomnia patients can be improved by pharmacological treatments $[4,5]$. So far as it is known, clinical efficacy of hypnotics is minimal for older people with insomnia and the risk of adverse events by using them, such as falls or cognitive impairment, is significantly increased [6]. For these reasons, nonpharmacologic treatments including exercise, cognitive behavior therapy for insomnia (CBT-I), and bright light 
therapy are suggested for chronic insomnia [7]. Although CBTI has been well known to be effective for improving total sleep time (TST) and sleep efficiency (SE), it might have little merit in terms of cost. Exercise has been proposed as an alternative for treating insomnia [8]. Previous studies indicate that participating physical activity or regularly exercising can improve subjective and objective sleep quality, and they can also have positive effects on mood and cognitive abilities $[9,10]$. Conversely, a study reported that poorer objective sleep quality was associated with poorer physical activity in older adults [11].

Sleep is regulated by homeostatic and circadian processes [10]. Circadian rhythms can be synchronized to external time cues called zeitgebers, which include light, timing of meals, physical activity and exercise [12]. In addition to its potent circadian regulation, exercise can be associated with changes in various physiological markers including sleep and hormone [10]. A few studies specifically evaluated the effect of exercise on sleep quality and sleep duration, implicating that exercise can overall contribute to improve the sleep quality $[7,8,13]$. However, previous studies have shown inconsistent findings regarding the relationship between exercise and sleep. A study found the reciprocal relationship between amount of physical activity and subjective sleep quality [14], but other studies did not [15]. Those inconsistencies may be due to a difference in the intensity or duration of exercise, or in the demographic characteristics across studies.

In general, sleep hygiene guidelines recommend avoiding exercising close to bedtime, for altered circadian rhythms depending on exercise timing can influence sleep negatively [6]. Youngstedt et al. [16] reported that the duration and time of variables related to exercise were the most consistent moderator, by which TST, slow wave sleep (SWS), REM sleep, and REM latency were significantly affected. The degree of light exposure should be considered as another potential factor regarding the effect of exercise on sleep. It has been inferred that the improvement of sleep quality through outdoor exercise with light exposure could be influenced by light exposure rather than by exercise itself [17]. A study applied non-pharmacological different strategies for chronic insomnia patients showed that light treatment group had a greater improvement in TST compared to exercise treatment group [18]. Passoss et al. [19], who conducted polysomnography on insomnia patients, found the significant reduction of sleep latency (SL) and wake time after sleep onset (WASO) and enhancement of SE after 6 months of aerobic exercise, suggesting aerobic exercise can have a positive effect on sleep. This study also reported the improved QOL and reduced mood symptoms like anxiety or depression following exercise. Most previous studies have investigated the effect of exercise on sleep through an intervention protocol focusing on insomnia patients or athletes. Thus, there was a limit to understanding the effect of exercise on sleep in general adults.

First, we aimed to compare the differences in subjective sleep quality, objective sleep parameters, and QOL according to the exercise duration in community-dwelling adults. Second, we were to compare the subjective sleep quality and objective sleep parameters between the normal control (NC) group, and the insomnia group with exercise and insomnia group without exercise. Third, we were to compare differences in subjective sleep quality and objective sleep parameters between the morning exercise group (EG) and afternoon EG, and those between the morning outdoor EG and afternoon outdoor EG, respectively.

\section{METHODS}

\section{Subjects}

This study was conducted by Kangwon National University Hospital Sleep Center from September 2013 to October 2015, on adults over 18 years of age who visited to the Hongcheongun, Hoengseong-gun, and Hwacheon-gun Health Centers in Gangwon-do. The questionnaires including the Korean version of Morningness-Eveningness Questionnaire (MEQ-K) [20], Pittsburgh Sleep Quality Index (PSQI) [21], Korean version of Epworth Sleepiness Scale (KESS) [22], Hyperarousal Scale (HAS) [23], Beck Depression Inventory (BDI) [24], and Short Form-12 Health Survey Questionnaire (SF-12) [25] were administered to each participant in addition to getting the demographic data like age, gender and education. In addition, questions about exercise such as the duration and timing of exercise as well as the place of exercise (outdoor or indoor).

The diagnosis for insomnia group followed the International Classification of Sleep Disorder, 2nd edition, 2005 (ICSD-2), using questions to recall their experiences for the past month regarding difficulty initiating sleep, difficulty maintaining sleep, early morning awakening, and non-restorative sleep. Subjects who responded "often" (3-4 days/week) or "almost every day" (more than 5 days/week) to one or more questions were included into the insomnia group. We excluded the subjects with irregular sleep-wake cycle due to shift work, those with the diagnoses of major depressive disorder, alcohol dependence or other substance abuse, and those with sleep disorders other than insomnia, according to the Diagnostic and Statistical Manual of Mental Disorder, 4th edition, Text Revision (DSM-IV-TR) [26]. We also excluded those who were taking hypnotics and had a history of cerebrovascular disease or an evidence of other neurological disorders, and those with cardiovascular disease, chronic pulmonary disease, chronic renal disease, malignant tumor, or uncontrollable diabetes or hypertension. When any subjects scored 12 or above on KESS, or 20 or above on BDI, phone interviews by a psychiatrist were conducted to exclude them from the study. Among 257 participants, 139 insomnia patients (age: $64.2 \pm 11.4 \mathrm{yr}$ ) and $84 \mathrm{NC}$ subjects (age $56.2 \pm 13.9 \mathrm{yr}$ ) were included in our final analysis.

The study protocol was approved by the Institutional Review 
Boardat Kangwon National University Hospital (2013-06-010017). Written informed consent was obtained from each enrolled participant. All procedures were performed in accordance with the principles outlined in the Declaration of Helsinki.

\section{Sleep Parameter Analysis}

All eligible participants wore Actiwatch-2 (Philips Respironics, Murrysville, PA, USA) for 7 days beginning at 6 p.m. on the initial day of measurement, and completed a sleep dairy during this time. For actigraphy, 1 sampling epoch was set to $1 \mathrm{~min}$, and Actiwatch Communication and Sleep Analysis Software (Actiware version 6.0.2, Philips Respironics) were used. Automated analysis by the software was used to obtain the results for night sleep parameters, including time in bed (TIB), TST, SL, SE, WASO, and fragmentation index (FI) which quantifies physical activity during sleep. Of the final sample 223 subjects who completed the survey, sleep parameters of 183 subjects were analyzed after excluding 15 who refused to wear the actigraph, 13 whose data were not recorded due Actiwatch malfunction, battery depletion, or software error, 11 who did not complete or incorrectly completed the sleep diary, and 1 with excessive duration of not wearing the equipment during the measurement period.

\section{Statistical Analysis}

Demographic characteristics, sleep need and caffeine intake of the EG and non-exercise group (non-EG) were compared using the t-test or $\chi^{2}$-test. Analysis of covariance (ANCOVA) adjusting for BDI was done to compare all questionnaires.

Subjects were divided according to the exercise duration into the non-EG, EG with 60 minutes or less, and EG with more than 60 minutes. ANCOVA was used to compare the scores of sleeprelated questionnaires and sleep parameters. Bonferroni correction was used for post-hoc analysis. For the comparison of those between the NC group, insomnia group with exercise, and insomnia group without exercise, ANCOVA was also done.

According to the timing of exercise, the EG was categorized into the morning EG if their exercise was done before the noon, and into the afternoon EG if done after the noon. ANCOVA was conducted on the sleep-related questionnaire adjusting for BDI, and sleep parameters adjusting for MEQ-K and BDI. In addition, the same analysis was conducted for the morning EG and afternoon EG in the outdoor EG.

All statistical analyses were conducted using SPSS 18.0 version (SPSS Inc, Chicago, IL, USA), and statistical significance was determined using $\mathrm{p}$-value of less than 0.05 .

\section{RESULTS}

In this study, there were no significant differences in the mean age, gender, BDI score, sleep need, caffeine intake and the ratio
Table 1. Demographic and clinical data in the non-EG and EG

\begin{tabular}{|c|c|c|c|}
\hline & $\begin{array}{l}\text { Non-EG } \\
(\mathrm{n}=104)\end{array}$ & $\begin{array}{c}\text { EG } \\
(n=119)\end{array}$ & $\mathrm{p}$-value \\
\hline Age (year) & $61.6 \pm 13.3$ & $60.8 \pm 12.8$ & 0.62 \\
\hline Gender $(\mathrm{M}: \mathrm{F})^{\dagger}$ & $27: 77$ & $44: 75$ & 0.08 \\
\hline Education (year) & $8.7 \pm 4.6$ & $10.3 \pm 5.0^{*}$ & 0.01 \\
\hline $\mathrm{BDI}$ & $8.7 \pm 5.3$ & $7.9 \pm 5.4$ & 0.25 \\
\hline Sleep need $(\mathrm{h})$ & $7.2 \pm 1.5$ & $7.1 \pm 1.4$ & 0.58 \\
\hline Caffeine intake $(\%)^{\dagger}$ & $54(51.9)$ & $67(56.3)$ & 0.51 \\
\hline Insomnia diagnosis $(\%)^{\dagger}$ & $64(61.5)$ & $75(63.0)$ & 0.82 \\
\hline
\end{tabular}

Values are shown as mean \pm SD or ratio.

${ }^{*} \mathrm{p}<0.05$ (independent $\mathrm{t}$-test, ${ }^{\mathrm{t}} \chi^{2}$-test).

EG: exercise group, non-EG: non-exercise group, BDI: Beck Depression Inventory.

of insomnia diagnosis between the EG and non-EG, but the education level of the EG was significantly higher than the non-EG $(\mathrm{p}=0.01)$ (Table 1).

Comparing the non-EG, EG with 60 min or less and EG with more than $60 \mathrm{~min}$, the EG with more than $60 \mathrm{~min}$ showed significantly higher HAS and Physical Component Summary (PCS) than the non-EG or EG with 60 min or less $(\mathrm{p}<0.01$, p $<0.01)$, and the EG with 60 min or less showed significantly higher Mental Component Summary (MCS) than the non-EG or EG with more than $60 \mathrm{~min}(\mathrm{p}=0.02)$. There were no significant differences in the MEQ-K, KESS, and PSQI, as well as in bed time (BT), wake time (WT), mid sleep time (MST), TIB, TST, SL, SE, WASO and FI between the three groups (Table 2).

Comparing the NC group, insomnia group with exercise, and insomnia group without exercise, there was no difference in PSQI scores between the insomnia group with exercise and insomnia group without exercise. The KESS scores were significantly higher in the insomnia group with exercise than NC group $(\mathrm{p}=0.03)$, and the PCS was significantly higher in the insomnia group with exercise than insomnia group without exercise, but showed no difference when compared to the NC group ( $\mathrm{p}<$ $0.01)$. There were no significant differences across the three groups in the MEQ-K, HAS, or MCS (Table 3). Analyzing the sleep parameters of the three groups, MST was significantly earlier in the insomnia group than the NC group $(\mathrm{p}=0.01)$. The insomnia group differed significantly from the NC group in SE and WASO ( $p<0.01, \mathrm{p}=0.01$ ), and these variables did not differ between the insomnia group with exercise and insomnia group without exercise. There were no significant differences across the three groups in BT, WT, TIB, TST, SL, or FI (Table 3).

The morning EG scored higher on the MEQ-K than the afternoon EG ( $\mathrm{p}<0.01$ ), and the two groups did not differ significantly on the KESS, PSQI, HAS, PCS, or MCS (Fig. 1A).

Moreover, in the case of outdoor exercise, the morning EG scored significantly higher on the MEQ-K $(\mathrm{p}=0.01)$, and the afternoon EG scored significantly lower on PSQI and significant- 
Table 2. Comparisons of the questionnaire scores and sleep parameters in the non-EG, and the $E G$ with $60 \mathrm{~min}$ or less (EG $\leq 60 \mathrm{~min} / \mathrm{day}$ ) and $E G$ with more than $60 \mathrm{~min}(E G>60 \mathrm{~min} /$ day $)$

\begin{tabular}{|c|c|c|c|c|c|}
\hline & Non-EG $(\mathrm{n}=104)$ & $\mathrm{EG} \leq 60 \min ^{\mathrm{b}}(\mathrm{n}=80)$ & $\mathrm{EG}>60 \min ^{\mathrm{c}}(\mathrm{n}=38)$ & $\mathrm{p}$-value & Bonferroni (post-hoc) \\
\hline \multicolumn{6}{|l|}{ Questionnaires } \\
\hline MEQ-K & $59.8 \pm 8.5$ & $59.7 \pm 9.9$ & $60.1 \pm 9.8$ & 0.96 & \\
\hline KESS & $5.5 \pm 3.0$ & $5.9 \pm 3.0$ & $5.1 \pm 3.8$ & 0.42 & \\
\hline PSQI & $8.0 \pm 4.3$ & $7.7 \pm 4.2$ & $8.2 \pm 4.0$ & 0.68 & \\
\hline HAS & $23.6 \pm 9.3$ & $25.6 \pm 9.3$ & $30.2 \pm 10.2$ & $<0.01^{\dagger}$ & $\mathrm{a}=\mathrm{b}<\mathrm{c}$ \\
\hline \multicolumn{6}{|l|}{ SF-12 } \\
\hline PCS & $39.0 \pm 12.2$ & $42.2 \pm 10.1$ & $45.9 \pm 7.9$ & $<0.01^{\dagger}$ & $\mathrm{a}<\mathrm{c}$ \\
\hline MCS & $53.6 \pm 8.9$ & $55.3 \pm 6.6$ & $51.4 \pm 7.3$ & $0.02^{*}$ & $b>c$ \\
\hline Sleep parameters & $\mathrm{n}=84$ & $\mathrm{n}=69$ & $\mathrm{n}=30$ & & \\
\hline Bedtime (h:m) & $22: 42 \pm 1: 13$ & $22: 38 \pm 1: 06$ & $22: 38 \pm 0: 55$ & 0.93 & \\
\hline Wake time (h:m) & $06.29 \pm 1.00$ & $06: 25 \pm 0.53$ & $06: 19 \pm 1: 13$ & 0.75 & \\
\hline Midsleep time (h:m) & $02: 45 \pm 0: 55$ & $02: 41 \pm 0: 51$ & $02: 37 \pm 0: 57$ & 0.26 & \\
\hline TIB (h) & $7.8 \pm 1.2$ & $7.8 \pm 1.1$ & $7.7 \pm 1.1$ & 0.91 & \\
\hline TST (h) & $6.2 \pm 1.0$ & $6.2 \pm 1.0$ & $6.0 \pm 1.0$ & 0.74 & \\
\hline $\mathrm{SL}(\min )$ & $17.9 \pm 15.9$ & $18.6 \pm 12.5$ & $17.9 \pm 12.2$ & 0.94 & \\
\hline SE (\%) & $78.7 \pm 9.6$ & $77.5 \pm 10.5$ & $78.5 \pm 6.1$ & 0.73 & \\
\hline WASO (min) & $58.8 \pm 25.5$ & $59.9 \pm 19.9$ & $57.6 \pm 21.7$ & 0.90 & \\
\hline FI & $31.2 \pm 10.7$ & $32.5 \pm 13.7$ & $30.9 \pm 9.5$ & 0.71 & \\
\hline
\end{tabular}

Values are shown as mean \pm SD.

${ }^{*} \mathrm{p}<0.05 .{ }^{\dagger} \mathrm{p}<0.01$ (ANCOVA controlling for Beck Depression Inventory).

EG: exercise group, non-EG: non-exercise group, MEQ-K: Korean version of Morningness-Eveningness Questionnaire, KESS: Korean version of Epworth Sleepiness Scale, PSQI: Pittsburgh Sleep Quality Index, HAS: Hyperarousal Scale, SF-12: Short Form-12 Health Survey Questionnaire, PCS: Physical Component Summary, MCS: Mental Component Summary, TIB: time in bed, TST: total sleep time, SL: sleep latency, SE: sleep efficiency, WASO: wake time after sleep onset, FI: fragmentation index.

ly higher on the KESS $(\mathrm{p}<0.05, \mathrm{p}=0.04)$. The two groups did not differ significantly on the HAS, PCS, or MCS (Fig. 1B).

There were no significant differences between the morning EG and afternoon EG in objective sleep parameters. In the case of outdoor exercise, there were also no significant differences between the morning EG and afternoon EG in objective sleep parameters (Table 4).

\section{DISCUSSION}

In this study, while there were no significant differences in age and gender between the EG and non-EG, the duration of education was significant higher in the EG (Table 1). In general, studies have shown that as the occupational, educational and income levels increase, subjective health status and health behaviors such as exercise also increase [27], and this study shows the same results.

In this study, no significant differences were observed in insomnia diagnosis rate and sleep need between the EG and nonEG (Table 1). In general, regular exercise has a positive effect on improvement of sleep disorder, and the frequency of insomnia is about $40 \%$ lower for those who engage in relatively large amounts of physical activity compared to those who do not [28]. In addition, although studies have shown that frequency of sleep disorder is lower for adults who exercise at least three hours a week [29], since these studies are cross-sectional studies, it is limited to conclude the causal relationship between exercise and sleep.

In this study, subjective sleep quality based on PSQI scores and objective sleep parameters did not differ significantly among the three groups by exercise duration (Table 2). Previous studies on exercise varied in the study design including the participants' age and health status, as well as the type, intensity or duration of exercise, showing inconsistent study results [14,30-32]. Several studies assessing the effect of aerobic exercise in adults have demonstrated positive benefits of exercise to sleep quality. A study for 13 community dwelling older adults showed that acute exercise following fitness training decreased WASO and resulted in a 71\% increase in SWS during subsequent sleep in comparison with the nonexercise condition in spite of a relatively small sample size [31]. These findings suggested daily exercise might be beneficial for sleep continuity and depth in the elderly. Furthermore, positive changes in sleep architecture in- 
Table 3. Comparisons of the questionnaire scores and sleep parameters between the normal control group (NC group), and the insomnia group with exercise and insomnia group without exercise

\begin{tabular}{|c|c|c|c|c|c|}
\hline & $\begin{array}{l}\text { NC group }^{\mathrm{a}} \\
(\mathrm{n}=84)\end{array}$ & $\begin{array}{l}\text { Insomnia group with exercise }{ }^{\mathrm{b}} \\
\qquad(\mathrm{n}=75)\end{array}$ & $\begin{array}{l}\text { Insomnia group without exercise }{ }^{c} \\
\qquad(\mathrm{n}=64)\end{array}$ & p-value & $\begin{array}{l}\text { Bonferroni } \\
\text { (post-hoc) }\end{array}$ \\
\hline \multicolumn{6}{|l|}{ Questionnaires } \\
\hline MEQ-K & $58.2 \pm 9.9$ & $61.0 \pm 9.0$ & $60.6 \pm 8.2$ & 0.23 & \\
\hline KESS & $6.3 \pm 3.0$ & $5.3 \pm 3.4$ & $5.0 \pm 3.1$ & $0.03^{*}$ & $a>c$ \\
\hline PSQI & $4.9 \pm 2.7$ & $9.6 \pm 3.7$ & $10.0 \pm 3.8$ & $<0.01^{\dagger}$ & $\mathrm{a}<\mathrm{b}=\mathrm{c}$ \\
\hline HAS & $23.4 \pm 8.9$ & $27.8 \pm 10.3$ & $25.3 \pm 9.4$ & 0.06 & \\
\hline \multicolumn{6}{|l|}{ SF-12 } \\
\hline PCS & $44.2 \pm 10.3$ & $42.4 \pm 9.2$ & $36.4 \pm 12.4$ & $<0.01^{\dagger}$ & $a=b>c$ \\
\hline MCS & $54.2 \pm 8.2$ & $52.9 \pm 7.5$ & $54.1 \pm 8.6$ & 0.60 & \\
\hline Sleep parameters & $\mathrm{n}=72$ & $\mathrm{n}=61$ & $\mathrm{n}=50$ & & \\
\hline Bedtime (h:m) & $22: 54 \pm 1: 10$ & $22: 29 \pm 1: 02$ & $22: 31 \pm 1: 07$ & 0.06 & \\
\hline Wake time (h:m) & $06: 36 \pm 1: 04$ & $06.17 \pm 0: 59$ & $6.21 \pm 0: 55$ & 0.07 & \\
\hline Midsleep time (h:m) & $02: 55 \pm 1: 00$ & $02: 33 \pm 0: 48$ & $02: 36 \pm 0: 50$ & $0.01^{*}$ & $a>b=c$ \\
\hline TIB (h) & $7.7 \pm 1.1$ & $7.8 \pm 1.2$ & $7.8 \pm 1.2$ & 0.92 & \\
\hline TST (h) & $6.3 \pm 0.9$ & $6.1 \pm 1.1$ & $6.1 \pm 1.0$ & 0.32 & \\
\hline $\mathrm{SL}(\min )$ & $16.0 \pm 9.5$ & $19.2 \pm 14.0$ & $19.9 \pm 18.8$ & 0.55 & \\
\hline SE (\%) & $81.4 \pm 5.7$ & $76.2 \pm 10.8$ & $76.2 \pm 11.0$ & $<0.01^{\dagger}$ & $a>b=c$ \\
\hline WASO (min) & $52.2 \pm 20.1$ & $63.5 \pm 21.1$ & $63.5 \pm 25.9$ & $0.01^{*}$ & $\mathrm{a}<\mathrm{b}=\mathrm{c}$ \\
\hline FI & $29.4 \pm 11.7$ & $33.2 \pm 11.2$ & $33.0 \pm 11.1$ & 0.08 & \\
\hline
\end{tabular}

Values are shown as mean \pm SD.

${ }^{*} \mathrm{p}<0.05 .{ }^{\dagger} \mathrm{p}<0.01$ (ANCOVA controlling for Beck Depression Inventory).

MEQ-K: Korean version of Morningness-Eveningness Questionnaire, KESS: Korean version of Epworth Sleepiness Scale, PSQI: Pittsburgh Sleep Quality Index, HAS: Hyperarousal Scale, SF-12: Short Form-12 Health Survey Questionnaire, PCS: Physical Component Summary, MCS: Mental Component Summary, TIB: time in bed, TST: total sleep time, SL: sleep latency, SE: sleep efficiency, WASO: wake time after sleep onset, FI: fragmentation index.

cluding SE have been found for adolescents as well as the elderly [33,34]. A large epidemiological study also showed that inactive participants had a higher risk of incident insomnia, highlighting the importance of physical activity to avoid insomnia among adults [32].

There was no significant difference in KESS scores as well as PSQI scores (Table 2). In general, daytime sleepiness can be a consequence of disturbed nocturnal sleep. It would be more plausible to show no group-difference in the severity of sleepiness as well as nocturnal sleep parameters in our study.

However, in this study, the EG with more than 60 min showed significantly higher HAS than the non-EG or EG with less than $60 \mathrm{~min}$ (Table 2). Hyperarousal is a key component in insomnia disorder, and cortisol level can be measured as a marker reflecting physiological hyperarousal [35]. A previous study showed the swimmers with overtraining had elevated cortisol levels, implicating that excessive exercise may lead to increased physiological hyperarousal [36]. Thus, our finding suggests subjects with exercise exceeding $60 \mathrm{~min}$ have a higher hyperarousal than those with exercise below $60 \mathrm{~min}$. Nevertheless, further study is required to investigate the difference in physiological markers reflecting hyperarousal by exercise duration in order to clarify this possibility.

In our study, it is likely that the longer exercise was associated with the higher level of arousal, irrespective of changes in the daytime sleepiness.

In this study, PCS scores were significantly higher in the EG with more than $60 \mathrm{~min}$, while MCS scores were significantly higher in the EG with less than 60 min than the EG with more than $60 \mathrm{~min}$ (Table 2). Wendel-Vos et al. [37] reported that physical QOL is improved through exercise in a general population, supporting our result that the physical QOL was significantly higher in subjects with exercise exceeding $60 \mathrm{~min}$ than in those with no exercise or exercise below $60 \mathrm{~min}$. However, our study showed that the mental QOL in subjects with exercise of longer duration exercise was worse compared to that in subjects with exercise of shorter duration exercise. A previous study reported depression was significantly worsened during overtraining in swimmers [36], suggesting excessive exercise may lead to increased psychological distress.

Through an additional analysis to investigate the relationship of sleep quality with QOL in our study, we found PSQI scores were negatively correlated with PCS scores $(r=-0.27, \mathrm{p}<0.01$, partial correlation analysis controlling for $\mathrm{BDI})$, but they were 


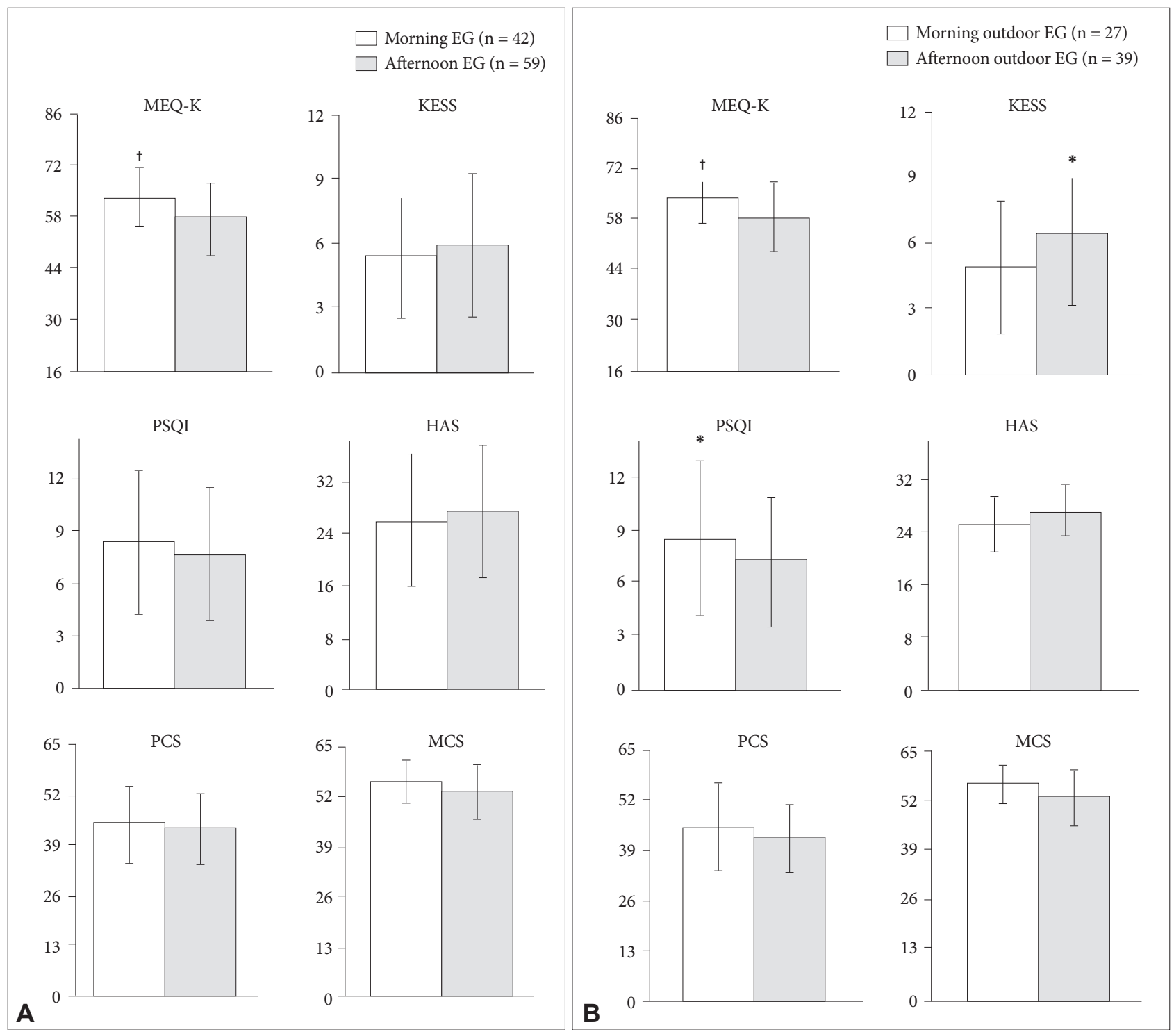

Fig. 1. Comparisons of the questionnaire scores between the morning exercise group (EG) and afternoon $E G$ ( $A$ ), and between the morning outdoor EG and afternoon outdoor EG (B). Error bars indicate mean \pm SD. ${ }^{*} p<0.05$. ${ }^{\dagger} p<0.01$ (ANCOVA controlling for Beck Depression Inventory). MEQ-K: Korean version of Morningness-Eveningness Questionnaire, KESS: Korean version of Epworth Sleepiness Scale, PSQI: Pittsburgh Sleep Quality Index, HAS: Hyperarousal scale, PCS: Physical Component Summary from Short Form-12 Health Survey Questionnaire (SF-12), MCS: Mental Component Summary from SF-12.

not significantly correlated with MCS scores. Although we found no difference in sleep quality among the three groups by exercise duration, this finding suggests that physical well-being, which was higher in our EG with more than $60 \mathrm{~min}$, can be associated with good sleep quality rather than psychological well-being.

This study showed no difference in PSQI scores and objective sleep parameters between the insomnia group with exercise and insomnia group without exercise (Table 3). Previous intervention studies have shown improvements in subjective sleep quality and objective sleep parameters after aerobic exercise of three times a week for 4 months or 6 months, based on exercise guidelines established for local residents complaining of insomnia [19,38], being different from our result. The type of exercise was not distinguished in this study, limiting the comparison of our result with previous ones. In this study, the KESS scores of the NC were significantly higher than those of insomnia group without exercise (Table 3). Although it may be expected that daytime sleepiness will occur to compensate for sleep deprivation in insomnia patients, it has been reported that daytime sleepiness may decrease due to the disrupted sleep homeostasis [39]. In this study, the degree of hyper-arousal indicated by the HAS was observed to be higher in the insomnia group than the NC group, although the difference was not statistically significant.

In this study, MST was significantly advanced in the insomnia group than the NC (Table 3). A previous study has reported 
Table 4. Comparisons of sleep parameters between the morning EG and afternoon EG, and those between the morning outdoor EG and afternoon outdoor EG

\begin{tabular}{|c|c|c|c|c|c|c|}
\hline & $\begin{array}{l}\text { Morning EG* } \\
\quad(\mathrm{n}=37)\end{array}$ & $\begin{array}{l}\text { Afternoon EG* } \\
\quad(\mathrm{n}=46)\end{array}$ & $\mathrm{p}$-value & $\begin{array}{l}\text { Morning outdoor EG* } \\
(\mathrm{n}=22)\end{array}$ & $\begin{array}{l}\text { Afternoon outdoor EG* } \\
\qquad(\mathrm{n}=32)\end{array}$ & $\mathrm{p}$-value \\
\hline TIB (h) & $7.8 \pm 1.1$ & $7.9 \pm 1.0$ & 0.59 & $7.9 \pm 1.2$ & $8.0 \pm 1.0$ & 0.86 \\
\hline TST (h) & $6.1 \pm 0.9$ & $6.3 \pm 0.9$ & 0.32 & $6.1 \pm 1.0$ & $6.4 \pm 1.0$ & 0.64 \\
\hline SL (min) & $16.4 \pm 9.3$ & $19.7 \pm 13.7$ & 0.38 & $16.6 \pm 9.3$ & $20.4 \pm 14.7$ & 0.26 \\
\hline SE (\%) & $77.9 \pm 5.1$ & $79.5 \pm 6.1$ & 0.36 & $77.6 \pm 4.6$ & $79.2 \pm 6.7$ & 0.74 \\
\hline WASO (min) & $61.8 \pm 19.2$ & $57.2 \pm 20.2$ & 0.57 & $61.0 \pm 18.3$ & $57.4 \pm 20.0$ & 0.95 \\
\hline FI & $32.0 \pm 9.5$ & $31.4 \pm 13.8$ & 0.56 & $31.7 \pm 8.8$ & $30.7 \pm 9.8$ & 0.66 \\
\hline
\end{tabular}

Values are shown as mean \pm SD. ANCOVA controlling for Beck Depression Inventory \& Korean version of Morningness-Eveningness Questionnaire.

*The morning EG and afternoon EG were defined as the EG before 12 pm and after 12 pm, respectively.

EG: exercise group, TIB: time in bed, TST: total sleep time, SL: sleep latency, SE: sleep efficiency, WASO: wake time after sleep onset, FI: fragmentation index.

that morning type becomes dominant as age increases after the age of 20 [40]. In this study, it could be interpreted that morning preference was shown in the insomnia group because the insomnia group were significantly older on average than the NC group. This study showed that PCS scores of the EG was significantly higher than those of the non-EG among insomnia patients (Table 3). Previous studies have shown a decrease in physical and mental QOL in insomnia patients, with impairment in physical domains especially prominent. [41,42] In addition, a previous study has reported that physical QOL increased in the insomnia group with exercise compared to the insomnia group without exercise [43], supporting the results of this study.

In this study, there was no difference in MCS scores according to whether they do exercise or not among insomnia patients (Table 3). While Passos et al. [19] reported an improvement in physical and mental QOL after aerobic exercise in insomnia patients, Reid et al. [43] reported that there was no change in mental QOL after exercise. In this study, there was no difference in subjective sleep quality and QOL between the morning and afternoon EG (Fig. 1A), and no difference in objective sleep parameters (Table 4). Previous research on insomnia patients reported no difference in subjective sleep quality, objective sleep parameters and QOL, when compared the effect of exercise for 6 months in the morning EG with that of the afternoon EG [19]. While most previous studies on exercise timing have reported that morning (7 AM) exercise is beneficial for improving overall sleep quality as acute exercise effects on sleep [44], there was a report that afternoon exercise increases sleep time and improve sleep quality in insomnia patients [6].

This study demonstrated that there was a significant decrease in the PSQI and increase in the KESS in the afternoon outdoor EG compared to the morning outdoor EG (Fig. 1B). Previous studies have reported that continuous exposure to bright light improves sleep [45] and the effect of light exposure on sleep is stronger than the effect of exercise on sleep [18]. In general, exercise in late afternoon is recommended to improve sleep hy- giene [6]. This study showed no difference in sleep parameters between the morning outdoor EG and afternoon outdoor EG (Table 4). Murray et al. [17] reported that, when engaging in outdoor exercise, SE improved in the morning outdoor EG, and that light exposure has potential effect on exercise levels and sleep quality. However, this study examined an acute effect of exercise, which would be different from ours. In outdoor exercise, the effect by the type or intensity of physical activity must be considered in addition to light exposure. Therefore, this study is limited in explaining the changes in objective sleep parameters due to only the timing of outdoor exercise.

The followings are limitations of this study. First, this study was conducted for rural residents only, so it is difficult to generalize the results to the entire population including urban residents. Second, this study included community-dwelling insomnia patients of relatively mild degree rather than insomnia patients who visited the hospital, resulting in the possibility that the differences in subjective sleep quality, objective sleep parameters, and QOL from exercise are not prominent. Third, this study may be limited in explaining the changes in sleep parameters because of not taking into account the effect of physical activity itself in addition to light exposure through outdoor exercise. Despite these limitations, this study is meaningful in that it attempted to examine the relationship of exercise with subjective sleep quality and objective sleep parameters, across the duration and timing of exercise in community-dwelling adults.

In this study, there were no differences in subjective sleep quality and objective sleep parameters across exercise duration, but physical QOL was higher when exercising more than 60 minutes during the day and metal QOL higher when exercising less than 60 minutes. Physical QOL was higher in the insomnia group with exercise as well. In addition, subjective sleep quality was higher in the afternoon outdoor EG when engaging in outdoor exercise. 


\section{Acknowledgments}

We thank our study participants in Gangwon-do, Republic of Korea. We are also grateful to public health professionals at the Hoengseong-gun, Hongcheon-gun and Hwacheon-gun public health centers who provided administrative support.

Support for this work was provided by Basic Science Research Program through the National Research Foundation of Korea (NRF) funded by the Ministry of Education, Science and Technology (2013R1A1A2009888).

\section{Conflicts of Interest}

The authors have no financial conflicts of interest.

\section{Authors' Contribution}

Conceptualization: Lee JH. Data curation: Bang JW. Formal analysis: Lee SH, Kim SJ. Funding acquisition: Lee JH. Methodology: Lee SH, Kim SJ, Lee JH. Project administration: Kim SJ. Supervision: Lee JH. Writingoriginal draft: Lee SH. Writing_review \& editing: Kim SJ, Lee JH.

\section{REFERENCES}

1. Ohayon MM. Epidemiology of insomnia: what we know and what we still need to learn. Sleep Med Rev 2002;6:97-111.

2. Hong SB. Sleep disorders medicine. J Korean Med Assoc 2013;56:410-22.

3. Kelley GA, Kelley KS. Exercise and sleep: a systematic review of previous meta-analyses. J Evid Based Med 2017;10:26-36.

4. Smith MT, Perlis ML, Park A, Smith MS, Pennington J, Giles DE, et al. Comparative meta-analysis of pharmacotherapy and behavior therapy for persistent insomnia. Am J Psychiatry 2002;159:5-11.

5. Glass J, Lanctôt KL, Herrmann N, Sproule BA, Busto UE. Sedative hypnotics in older people with insomnia: meta-analysis of risks and benefits. BMJ 2005;331:1169.

6. Morin CM, Mimeault V, Gagné A. Nonpharmacological treatment of late-life insomnia. J Psychosom Res 1999;46:103-16.

7. Montgomery P, Dennis J. A systematic review of non-pharmacological therapies for sleep problems in later life. Sleep Med Rev 2004;8:47-62.

8. Montgomery P, Dennis J. Cognitive behavioural interventions for sleep problems in adults aged 60+. Cochrane Database Syst Rev 2003;1:CD 003161.

9. de Castro Toledo Guimaraes LH, de Carvalho LB, Yanaguibashi G, do Prado GF. Physically active elderly women sleep more and better than sedentary women. Sleep Med 2008;9:488-93.

10. Buman MP, King AC. Exercise as a treatment to enhance sleep. Am J Lifestyle Med 2010;4:500-14.

11. Dam TT, Ewing S, Ancoli-Israel S, Ensrud K, Redline S, Stone K; Osteoporotic Fractures in Men Research Group. Association between sleep and physical function in older men: the osteoporotic fractures in men sleep study. J Am Geriatr Soc 2008;56:1665-73.

12. Klein DC, Moore RY, Reppert SM. Suprachiasmatic nucleus: the mind's clock. New York: Oxford University Press 1991;125-43.

13. Yang PY, Ho KH, Chen HC, Chien MY. Exercise training improves sleep quality in middle-aged and older adults with sleep problems: a systematic review. J Physiother 2012;58:157-63.

14. Dzierzewski JM, Buman MP, Giacobbi PR Jr, Roberts BL, Aiken-Morgan AT, Marsiske M, et al. Exercise and sleep in community-dwelling older adults: evidence for a reciprocal relationship. J Sleep Res 2014;23: 61-8.

15. Mitchell JA, Godbole S, Moran K, Murray K, James P, Laden F, et al. No evidence of reciprocal associations between daily sleep and physical activity. Med Sci Sports Exerc 2016;48:1950-6.

16. Youngstedt SD, O'Connor PJ, Dishman RK. The effects of acute exercise on sleep: a quantitative synthesis. Sleep 1997;20:203-14.

17. Murray K, Godbole S, Natarajan L, Full K, Hipp JA, Glanz K, et al. The relations between sleep, time of physical activity, and time outdoors among adult women. PLoS One 2017;12:e0182013.

18. Guilleminault C, Clerk A, Black J, Labanowski M, Pelayo R, Claman D.
Nondrug treatment trials in psychologic insomnia. Ann Intern Med 1995; 155:838-44.

19. Passos GS, Poyares D, Santana MG, D’Aurea CV, Youngstedt SD, Tufik $\mathrm{S}$, et al. Effects of moderate aerobic exercise training on chronic primary insomnia. Sleep Med 2011;12:1018-27.

20. Lee JH, Kim SJ, Lee SY, Jang KH, Kim IS, Duffy JF. Reliability and validity of the Korean version of Morningness-Eveningness Questionnaire in adults aged 20-39 years. Chronobiol Int 2014;31:479-86.

21. Buysse DJ, Reynolds CF 3rd, Monk TH, Berman SR, Kupfer DJ. The Pittsburgh Sleep Quality Index: a new instrument for psychiatric practice and research. Psychiatry Res 1989;28:193-213.

22. Cho YW, Lee JH, Son HK, Lee SH, Shin C, Johns MW. The reliability and validity of the Korean version of the Epworth sleepiness scale. Sleep Breath 2011;15:377-84.

23. Pavlova M, Berg O, Gleason R, Walker F, Roberts S, Regestein Q. Selfreported hyperarousal traits among insomnia patients. J Psychosom Res 2001;51:435-41.

24. Lee YH, Song JY. A study of the reliability and the validity of the BDI, SDS, and MMPI-D scales. Kor J Clin Psychol 1991;10:98-113.

25. Ware J Jr, Kosinski M, Keller SD. A 12-Item Short-Form Health Survey: construction of scales and preliminary tests of reliability and validity. Med Care 1996;34:220-33.

26. American Psychiatric Association. Diagnostic and Statistical Manual of Mental Disorders (DSM-IV). 4th ed. Washington, DC: American Psychiatric Association 2000.

27. Idler EL, Benyamini Y. Self-rated health and mortality: a review of twenty-seven community studies. J Health Soc Behav 1997;38:21-37.

28. Peppard PE, Young T. Exercise and sleep-disordered breathing: an association independent of body habitus. Sleep 2004;27:480-4.

29. Quan SF, O'Connor GT, Quan JS, Redline S, Resnick HE, Shahar E, et al. Association of physical activity with sleep-disordered breathing. Sleep Breath 2007;11:149-57.

30. Yamanaka Y, Hashimoto S, Takasu NN, Tanahashi Y, Nishide SY, Honma S, et al. Morning and evening physical exercise differentially regulate the autonomic nervous system during nocturnal sleep in humans. Am J Physiol Regul Integr Comp Physiol 2015;309:R1112-21.

31. Melancon MO, Lorrain D, Dionne IJ. Sleep depth and continuity before and after chronic exercise in older men: electrophysiological evidence. Physiol Behav 2015;140:203-8.

32. Chen LJ, Steptoe A, Chen YH, Ku PW, Lin CH. Physical activity, smoking, and the incidence of clinically diagnosed insomnia. Sleep Med 2017;30:189-94.

33. Gambassi BB, Almeida FJ, Sauaia BA, Novais TM, Furtado AE, Chaves LF, et al. Resistance training contributes to variability in heart rate and quality of the sleep in elderly women without comorbidities. J Exerc Physiol Online 2015;18:112-23.

34. Mendelson M, Borowik A, Michallet AS, Perrin C, Monneret D, Faure $\mathrm{P}$, et al. Sleep quality, sleep duration and physical activity in obese adolescents: effects of exercise training. Pediatr Obes 2016;11:26-32.

35. Kalmbach DA, Cuamatzi-Castelan AS, Tonnu CV, Tran KM, Anderson JR, Roth T, et al. Hyperarousal and sleep reactivity in insomnia: current insights. Nat Sci Sleep 2018;10:193-201.

36. O'Connor PJ, Morgan WP, Raglin JS, Barksdale CM, Kalin NH. Mood state and salivary cortisol levels following overtraining in female swimmers. Psychoneuroendocrinology 1989;14:303-10.

37. Wendel-Vos GC, Schuit AJ, Tijhuis MA, Kromhout D. Leisure time physical activity and health-related quality of life: cross-sectional and longitudinal associations. Qual Life Res 2004;13:667-77.

38. Baron KG, Reid KJ, Zee PC. Exercise to improve sleep in insomnia: exploration of the bidirectional effects. J Clin Sleep Med 2013;9:819-24.

39. Lim YC, Kim SJ, Kwon HJ, Lee JH. The effects of stress- and sleep-related variables on the quality of life in insomnia patients. J Korean Neuropsychiatr Assoc 2016;55:277-85.

40. Paine SJ, Gander PH, Travier N. The epidemiology of morningness-eveningness: influence of age, gender, ethnicity, and socioeconomic factors 
in adults (30-49 years). J Biol Rhythms 2006;21:68-76.

41. Zammit GK, Weiner J, Damato N, Sillup GP, McMillan CA. Quality of life in people with insomnia. Sleep 1999;22 Suppl 2:S379-85.

42. Stein MB, Belik SL, Jacobi F, Sareen J. Impairment associated with sleep problems in the community: relationship to physical and mental health comorbidity. Psychosom Med 2008;70:913-9.

43. Reid KJ, Baron KG, Lu B, Naylor E, Wolfe L, Zee PC. Aerobic exercise improves self-reported sleep and quality of life in older adults with in- somnia. Sleep Med 2010;11:934-40.

44. Fairbrother K, Cartner B, Alley JR, Curry CD, Dickinson DL, Morris $\mathrm{DM}$, et al. Effects of exercise timing on sleep architecture and nocturnal blood pressure in prehypertensives. Vasc Health Risk Manag 2014;10: 691-8.

45. Campbell SS, Dawson D, Anderson MW. Alleviation of sleep maintenance insomnia with timed exposure to bright light. J Am Geriatr Soc 1993;41:829-36. 